\title{
La popularité des "Evangiles des quenouilles": un paradoxe révélateur
}

\author{
MADELEINE JEAY
}

Une étude de la réception des Evangiles des Quenouilles à la fin du XVe et au début du XVIe siècle apporte plus qu'une simple preuve de leur popularité. Elle révèle une attitude paradoxale à leur égard et par cela pose un problème d'ordre plus général. On constate en effet qu'alors même que les Evangiles des Quenouilles jouissaient d'une exceptionnelle popularité, ils se voyaient rejetés comme objet de mépris. Nous verrons, et ce sera le propos de cet article, que pour qui veut en comprendre la raison d'être, cette situation paradoxale témoigne des conflits qui ont permis au système de valeurs de la Renaissance de se constituer.

De la popularité des Evangiles des Quenouilles, on ne saurait douter: les indices habituels en la matière en font une évidence. L'oeuvre apparut sous forme manuscrite ${ }^{1}$ et connut, après l'édition qu'en fit Colard Mansion à Bruges vers $1480,{ }^{2}$ une dizaine d'éditions et de réimpressions, ainsi que des traductions (en anglais, néerlandais et allemand) et une adaptation versifiée en dialecte toulousain. ${ }^{3} \mathrm{Il}$ faut leur ajouter, comme éléments de preuve, les nombreuses mentions qui sont faites à notre oeuvre. Ce sont elles qui nous préoccupent plus spécifiquement ici, car elles illustrent bien le sort singulier qui fut réservé à ce texte. La référence aux Quenouilles n'arrive jamais au hasard, bien au contraire. Qu'on en juge. Lorsqu'elle ne tombe pas de la plume méprisante des moralistes, pédagogues ou censeurs, elle apparaît dans la bouche des sots, des fous, des paysans, ou survient à propos des femmes, de la femme acariâtre et luxurieuse de la farce. Opposition révélatrice, sans aucun doute, et qu'il est permis de formuler avec les notions empruntées à Mikhaïl Bakhtine, car elles conviennent particulièrement bien à notre cas. Le sérieux des moralistes affronte le monde du rire dans lequel les Evangiles des Quenouilles trouvent naturellement leur place, et le rejette faute de le comprendre. Il les fait basculer d'un contexte de rire et de folie dans celui de la sagesse et de la censure, et décrit ainsi les deux étapes de notre parcours.

Mais avant d'entreprendre celui-ci, une courte présentation des Evangiles des Quenouilles s'impose puisque c'est à peine s'ils commencent à sortir de 
la zone d'ombre et de silence où les critères de qualité de la critique dite littéraire les confinaient. ${ }^{4}$ Les Evangiles des Quenouilles distribuent en six veillées de fileuses une collection d'à peu près 250 croyances populaires. Cet hexaméron où une fileuse prend chaque soir la parole pour enseigner à ses compagnes les superstitions qu'elle connaît, emprunte la structure des histoires à journées dans la tradition de Boccace. Par manière de parodie, celle de la littérature scolaire, une auditrice souvent affublée d'un nom burlesque, apporte sa "glose": simple confirmation, croyance nouvelle ou commentaire ironique parfois axé sur un jeu de mots grivois. Un seul exemple suffira pour faire apprécier la teneur et la saveur de ces échanges:

Cellui qui franchement puet chevauchier l'ours .ix. pas d'un tenant, il est affranchy de .ix. paires de maladies.

Glose. Dist une vielle matrone qui derriere les autres estoit: Je cuide bien qu'il soit vray de la garison desdictes .ix. maladies, mais non pas de celles dont on chiet a la renverse. ${ }^{5}$

Les intérêts de nos fileuses touchent aux aspects essentiels de la vie: naissance, amour, mariage. Elles se centrent autour des préoccupations quotidiennes de l'homme et de la femme à propos de la santé et de la richesse, elles savent comment se présagent le bonheur et le malheur. Issu des milieux ruraux et traditionnellement transmis par voie orale, par la bouche des femmes, ce savoir est ici rapporté et mis en forme par un homme, un "secrétaire" qui ne se prive pas d'intervenir pour en rire et ironiser sur lui, dans le prologue, dans les transitions entre les journées et la conclusion.

Les éléments qui se révèlent comme constitutifs des Evangiles des Quenouilles, le rural et ses traditions, son oralité, le féminin et ses connotations au sexuel, à l'occulte, au diabolique, se retrouvent comme thèmes récurrents, inlassablement repris et modulés dans l'ensemble du corpus qui appartient au registre comique. A ce titre, il existe une affinité de fait, une identité consubstantielle entre notre oeuvre et celles où nous la verrons citée dans notre première série de textes. Sa présence y joue un double rôle. D'une part, elle accentue la tonalité comique du texte hôte; d'autre part, elle subsume sous le seul titre de Quenouilles, un faisceau de potentialités qui renvoient à cette tonalité. La citation acquiert de ce fait une haute valeur connotative et évoque à elle seule, l'univers du comique et certains de ses éléments constitutifs.

Nous verrons le processus à l'oeuvre dès notre premier exemple, le plus ancien: le Mystère de l'Incarnation et Nativité de Notre Sauveur et Rédempteur Jésus-Christ représenté à Rouen en $1474 .{ }^{6}$ Au cours de la deuxième journée, dans une scène de bergers destinée à "récréer joyeuse- 
ment l'esprit des auditeurs,"' Ludin, le personnage drôle du mystère se rappelle les veillées (sairies) où sa grand-mère contait et transmettait ce qu'elle avait lu au "livre des Quenouilles."8 Beau prétexte pour un jeu sur les rimes en "ouille"! Mais l'échange de répliques, à travers le jeu verbal, exprime le paradoxe inhérent à ce livre et qui se révèle dès la première référence qui en est faite. Elle situe en effet les Quenouilles dans le cadre qui leur est propre et qui les constitue, celui de la veillée, lieu de transmission privilégié de la culture folklorique rurale. D'emblée nous sommes projetés au coeur des problèmes que pose la situation de cette culture au XVe et au XVIe siècles, au confluent de l'oral et de l'écrit, entre une civilisation de la mémoire et de la parole, et celle qui verra l'imprimerie faire triompher la fixation des textes et leur connaissance par l'approche individuelle de la lecture. La citation laisse entrevoir un monde, celui d'une sagesse ancestrale transmise par la bouche des personnes âgées; elle signale le rôle de la femme comme médiatrice des savoirs ancestraux. Prise au pied de la lettre, elle nous laisse entendre qu'une situation de pure oralité tient de la fiction, déjà au XVe siècle. Elle nous montre la part faite au livre, même lorsque seul un petit nombre d'individus sait lire. On passe de la parole à la lecture, elles se combinent, se complètent, soit qu'une personne lise à voix haute pour un groupe d'auditeurs, soit qu'elle retransmette oralement ce qu'elle a appris dans la solitude d'une lecture individuelle.

Reste à comprendre pourquoi le témoignage de Ludin porte à rire, pourquoi les autres bergers se moquent de lui: "tu ne sces mais ou tu en es." Il faut voir là une distance ironique non seulement à l'égard des Evangiles des Quenouilles, mais plus globalement, à l'égard des coutumes populaires ainsi que de l'univers culturel du rural, de l'illettré. Se joint à cela, le thème de la vieille femme experte en recettes et superstitions de toutes sortes, commun à ce passage du Mystère et à l'oeuvre citée, thème que nous n'avons pas fini de rencontrer.

De l'intermède comique du mystère à la farce, le passage se fait de luimême. Nous plongeons alors au beau milieu d'une querelle domestique, celle de la face du Pont aux Asnes, ${ }^{9}$ prototype d'une des deux catégories de farces conjugales. ${ }^{10}$ Ici ce n'est pas un conflit sexuel qui oppose le mari et la femme, mais leur affrontement pour le pouvoir au sein du ménage. Impuissant face au désir de domination de sa femme, à son refus total d'obéir, le mari ne peut qu'alléguer le Livre des Quenouilles où est "récité en catholicon,"

Qu'il faut que nous vaincon

Et que les hommes soient les maistres. ${ }^{11}$

Piètre argument, et sans effet: elle ne mettra pas plus qu'avant les pois au pot. Il est vrai que nous chercherions vainement une telle assertion dans 
les Evangiles des Quenouilles. Ceux-ci ne prétendent-ils pas au contraire avoir été écrits "a l'onneur et exaucement des dames"! 12 Le contexte de la citation prend donc le contrepied de ce qui semble l'objectif de l'oeuvre citée. Il suffit pourtant de quitter le sens littéral pour se rendre compte que les deux oeuvres s'insèrent dans le même contexte intertextuel et se réfèrent au lieu commun littéraire de l'antiféminisme. Nos deux oeuvres se rejoignent, non pas en ce qu'elles prennent position pour l'un ou l'autre dans la querelle qui oppose les sexes, mais parce qu'elles jouent toutes deux sur ce thème. Elles laissent entrevoir toutes deux un monde d'hommes dominés, impuissants, de maris battus et cocus, ${ }^{13}$ un monde inversé par rapport à celui de la réalité, à l'ordre normal, celui d'une structure familiale patriarcale basée sur l'obéissance et la fidélité de la femme. Sur les tréteaux de la farce et autour d'eux, des hommes $s^{\prime}$ 'adressent à d'autres hommes ${ }^{14}$ et présentent la version ludique, comique, du rapport entre les sexes: elles s'exprime en termes d'inversion sexuelle. Et comme il s'agit de bourgeois cultivés, ${ }^{15}$ la distance sociale se superpose à l'altérité sexuelle. La femme, le berger Ludin et sa vieille grand-mère se retrouvent ensemble dans le monde à l'envers du rire où nous allons aussi rencontrer Gilette, l'ancienne prostituée, l'entremetteuse des Tromperies de Pierre de Larrivey. ${ }^{16}$

Elle aussi allègue les Quenouilles! Non contre un mari, mais contre sa fille, la courtisane Dorothée qui, amoureuse du jeune Constant répugne de plus en plus au métier que lui impose sa mère, et ne peut plus dissimuler son dégoût pour le médecin, un vieillard qui la paie en cadeaux et en argent. Cette bonne mère la traite de sotte et lui fait la leçon:

Bienheureuse est celle dont un vieil rassoté est amoureux!

Sçais-tu ce que dict une glose sur le chapitre troisième du livre des Quenouilles:

Au vieil rassoté fay caresses

Si en bref veux avoir richesses. ${ }^{17}$

Comme tout à l'heure, on nous renvoie à une autorité imaginaire. Non seulement cet énoncé ne se trouve pas dans les Evangiles des Quenouilles, mais on n'y traite aucunement des relations vénales entre des jeunes filles et des vieillards. Comme pour le Pont aux Asnes, l'affinité, la parenté entre les Evangiles des Quenouilles et Les Tromperies ne s'établit pas au niveau littéral du texte, mais à celui des références intertextuelles, soit à travers le personnage qui apparaît dans les deux oeuvres, l'entremetteuse. En effet, le portrait-type de la narratrice des Evangiles des Quenouilles correspond par bien des traits à celui de la maquerelle Gilette et plus généralement, à celui que La Célestine à la vogue si grande au XVIe siècle, a popularisé. ${ }^{18}$ Le modèle du personnage, codifié depuis longtemps, remonte le Moyen-âge jusqu'à l'Antiquité, jusqu'à 
l'aubergiste de l'Ane d'or d'Apulée, la vieille magicienne Méroé. ${ }^{19}$ L'image synchrétique qui se dégage de la description des six narratrices des Evangiles des Quenouilles nous montre une vieille femme remarquable par sa laideur et qui se définit par deux types d'activité: la sorcellerie et le sexe. A la sorcellerie, se rattachent ses talents de magicienne et de guérisseuse, talents qui lui confèrent le statut d'accoucheuse mais lui permettent aussi, sous ce masque, de pratiquer les avortements, refaire les virginités et concocter les philtres d'amour. Car le sexe est l'autre grande affaire de cette "marchande de luxure" ${ }^{20}$ qui vit difficilement du commerce de la prostitution. La citation apporte donc ici tout une charge référentielle, la gamme des allusions qu'elle porte en elle et qui surenchérissent sur le climat trouble mais comique de la scène.

Il faut supposer que des bourgeois lettrés comme ceux qui prenaient plaisir au spectacle des farces, pouvaient encore comprendre les caractères de la topique antiféministe dont la vieille maquerelle est un élément, et s'en amuser. C'est dire qu'ils ne se sentaient pas encore coupés de ce que Bakhtine appelle la tradition gauloise. On peut critiquer ce terme ainsi que la dichotomie qui l'oppose à celui de "tradition sérieuse," mais il faut reconnaître la fécondité de l'intuition de Bakhtine lorsqu'il voit dans la femme en contexte comique, non la victime de la misogynie masculine, mais une image ambivalente. Celle qui lui apparaît comme une "injure incarnée" et la "tombe corporelle de l'homme," se révèle aussi comme un inépuisable vaisseau de fécondation qui voue à la mort tout ce qui est vieux et achevé. ${ }^{21}$ Ses attaches avec le matériel, le bas corporel, l'obscène, rabaissants mais forces régénératrices, ne la vouent pas au négatif de façon univoque. Elles la situent du côté de l'irrationnel, de la folie, ambivalents tout autant qu'elle et composantes de base du registre comique. Nous avions trouvé les Evangiles des Quenouilles en corrélation avec le rusticus, le berger naïf des intermèdes comiques du mystère, avec la femme dominatrice et débauchée; ne nous étonnons pas maintenant de les rencontrer rattachés au contexte de la folie. Prenons ce passage du Sermon des Foulx ${ }^{22}$ :

Ergo donc, il y a plus de sotz

Que de saiges, c'est mon propos,

Y a il prou sotz, gloses et notes,

Encore y a il plus de sottes.

Qui vouldroit escrire des femmes,

Il fauldroit de papier dix rames;

Toute femme filant quenouille

Est plus sotte que n'est gribouille.

Certes, la première lecture ne révèle pas la moindre allusion aux Evangiles des Quenouilles. On y retrouve des termes propres à notre oeuvre, "glose" et "quenouilles," et la situation semblable où quelqu'un "vouldroit escrire 
des femmes.' Est-ce suffisant pour y reconnaître une allusion? On retiendra que les femmes objets d'écriture, les fileuses et leur quenouille sont associées à la folie. Un épisode de la Farce d'un savetier nommé Calbain ${ }^{23}$ pose le même problème et conduit aux mêmes conclusions. Aux requêtes de sa femme qui veut une robe, Calbain répond par des chansons. Mais elle l'enivre, l'endort et lui dérobe sa bourse, puis à son tour ne répond que par des refrains lorsque son mari veut reprendre son bien. Or, au moment où il se réveille et se rend compte du larcin dont il est victime, voilà Calbain qui s'écrie:

Ha, je suis tout enquenouillé

Et de mon bon sens fatrouillé. ${ }^{24}$

Autrement dit, les femmes rendent les hommes fous. L'allusion à notre ouvrage a beau ne pas être évidente, il faut reconnaître au terme "quenouille," un emploi métaphorique qui s'étend sans aucun doute à l'oeuvre elle-même et qui permet de désigner la folie. Mais écoutons le Fol dans une de ses tirades de la Vie et Passion de Monseigneur Saint Didier, mystère composé par le chanoine Guillaume Flamang et joué à Langres en $1482^{25}$ :
Voila doucement caqueté.
Dieu! Comme il y fait precieux!
Qui sont ces petits soupireux
Qui ont ailes comme coulons?
Sont-ce point ces blanc papillons
Qui cheminent sur des echasses?
Ils sont revestus de besaces
De tel couleur qu'on fait les brayes.
Je vous dis paroles plus vrayes
Que n'est le livre des Quelongnes. ${ }^{26}$

On voit le sens que prend cette référence venant après une suite d'absurdités de style fatrasique. On ne peut mieux établir l'analogie entre le contenu du livre et les propos tenus par le fou.

Or le fou ne se confine pas à une existence de thème littéraire: les bouffons de cour, "véhicules permanents du principe de carnaval dans la vie courante," selon Bakhtine, ${ }^{27}$ assurent la présence concrète de la notion d'inversion. Il était donc logique, après ce que nous avons dit sur l'appartenance des Evangiles des Quenouilles à l'univers inversé du rire, de les trouver dans le répertoire de maître Guillaume, fou de François Ier. ${ }^{28}$ "Par la folie, commence et finit la grande inversion." 29 C'est là, dans ce principe d'inversion, que la présence des Evangiles des Quenouilles dans les oeuvres comiques et dans le répertoire du fou de cour, trouve son explication. Soit: par leur appartenance commune au monde carnavalesque. ${ }^{30}$ Dans la béance du rire, se rejoignent tous ces personnages: le paysan naïf, le sot, le fou, la femme, tous plus ou moins liés avec le diable des mystères. La diablerie n'est-elle pas 
la part carnavalesque du mystère? Ne s'oppose-t-elle pas à ce qui represénte le bien, la raison, la sagesse? Lorsqu'elle sort dans la rue, elle se déploie en cortège de licence et de privautés analogues à celui du charivari ou du carnaval. ${ }^{31}$ L'outrance gestuelle et verbale caractérisent aussi bien l'intermède farcesque du mystère, la farce elle-même, et la sottie. D'où la crudité de leur langage marqué, comme celui du fou, par l'obscénité et la scatologie. ${ }^{32}$ Les formes littéraires du registre comique ne se dissocient pas de ses formes rituelles. Par leur caractère ludique ou carrément festif, elles appartiennent toutes à ce que Bakhtine appelle "la culture comique populaire." ${ }^{33}$ L'intérêt de ce concept, c'est qu'il fait ressortir sous la diversité des phénomènes, l'unité du système que $P$. Zumthor a désigné comme une "contre-culture," un "ordre du désordre." ${ }^{34}$ Ce monde du rire, du corporel ou de la folie, peu importe le terme par lequel on le désigne, ne s'oppose pas à l'autre, celui de la sagesse et du sérieux pour le contredire, mais pour le compléter. Ils coexistent pour donner une image du monde dichotomisée certes, mais totale, où l'irrationnel trouve sa place. C'est celui-ci qu'allégorisent les Evangiles des Quenouilles dans l'oeuvre où ils prennent place. Allégorie globale, elle voue à l'irrationnel le peuple paysan et sa culture, le rusticus toujours si proche de l'homme sauvage, la femme doublement impliquée: par sa sexualité, par les savoirs superstitieux qui la rapprochent de la sorcière.

La plupart de ces éléments thématiques et scéniques se trouvent accumulés dans le bref espace de la Lettre missive a venerable et cathefumineuse personne, Jo. de Wisoc, president en Papagosse de Jean Molinent où il s'agit de décrypter les "difficultés du Livre des Quenouilles.",35 On comprend $\mathrm{P}$. Champion de ne pas chercher à identifier le destinataire, ce "monstre pronosticateur" "36: c'est un véritable masque de carnaval que Molinet où il s'agit de décrypter les “difficultés du Livre des Quenouilles.",35 cohérence de l'univers du comique, il porte en lui l'occulte et l'insensé, l'obscène et le scatologique. ${ }^{37}$ Aux éléments thématiques du sorcier, "homme faé, divin séraph," du guérisseur et de l'astrologue faiseur de pronostications absurdes, s'ajoute sur le plan linguistique, le burlesque des constructions verbales imaginaires ${ }^{38}$ et des accumulations d'absurdités. ${ }^{39}$ Nous avions déjà rencontré les Quenouilles en contexte fatrasique, dans le monologue du fou du mystère de saint Didier. Mais ici, leur citation arrive en pleine scatologie, et ceci très littéralement:

votre pance est si enflee de lard de rientropicque qu'il vous eschappe bien souvent, Monseigneur, par derriere; pourquoi vos petis disciples, escoliers par decha, voeullans sucher la clere matiere resplandissant en vostre brun antiphonier, vous supplient a nudz genoux, lesquelz en bas, comme a leur pere, paterne, porron et patriarche, qu'il vous plaise desvoleper les tresoirs de vostre grand aulmaire et retourner les feulliés aux difficultés du Livre des Quenouilles, pourquoy ils puissent mordre tout ens, Monseigneur, jusques a braies. ${ }^{40}$ 
Faut-il conclure que le contenu des Quenouilles soit pour Molinet si vile matière et son jugement sur elles absolument négatif? Pensons plutôt qu'elles appartiennent dans son esprit au même registre que sa burlesque "lettre missive." Qu'elle figure un de ces gestes carnavalesques propres au géant Gaster du Quart Livre: "Ainsi Gaster renvoyait ces Matagotz à sa scelle persee veoir, considerer, philosopher et contempler quelle divinité ilz trouvoient en sa matiere fecale." ${ }^{41}$

C'est dans un contexte tout aussi scabreux que celui de Molinet, que Noël du Faïl cite les Quenouilles, à propos des souvenirs que racontent trois femmes à la vie douteuse et à la conversation leste, dans les Contes d'Eutrapel. $^{42}$ La constellation: Evangiles des Quenouilles, féminité et sexualité, que nous avons déjà rencontrée, réapparaît, structure décidément constitutive du registre comique. Encore une fois, qu'on ne cherche aucun rapport littéral entre la citation et l'oeuvre citée. Le lien, métaphorique, se fait au niveau de l'allusion, c'est-à-dire au type de matrones qui figurent dans les Evangiles des Quenouilles. L'une des trois conteuses se prétend "pour avoir fait fils et fille, et conséquemment, dit le livre des Quenoilles, licentiee de tout dire." ${ }^{\prime 43}$

Elles possèdent donc le type d'expérience que suppose l'acquisition du savoir que transmettent les Evangiles des Quenouilles: connaissance de l'amour et des hommes ainsi que tout ce qui concerne la grossesse et les enfants. On se situe toujours dans l'univers du rire et parmi ses composantes, avec celles qui se rattachent à la femme et à l'érotisme. C'est à titre de charge comique que N. du Faïl a lui aussi utilisé les Evangiles des Quenouilles dans les Contes d'Eutrapel. Par contre, l'usage qu'il en a fait dans les Propos rustiques apparaît comme beaucoup plus complexe et ambigu. Il les prend au sérieux et leur confie une valeur documentaire, de témoignage sur les usages et croyances des paysans. Aucun lien littéral ne rapproche les Propos rustiques des Evangiles des Quenouilles, si l'on excepte ce terme d' "agoubilles" qui leur est selon toute vraisemblance, emprunté. ${ }^{44}$ Mais leur trace se devine sans peine. Du Faill ne reprend textuellement aucune des superstitions des Quenouilles, il s'est simplement amusé à en faire un condensé lorsqu'il énumère les croyances du fils de Gobemouche. ${ }^{45}$ Il les rejoint dans son intérêt pour la vie rurale, pour les paysans et leurs traditions. Cet intérêt ne va pas sans ambigüité: dans les Contes d'Eutrapel, il éprouve le besoin de faire intervenir Polygame en faveur de la vraie religion et contre les croyances superstitieuses. ${ }^{46}$ De la même façon, son éloge de la vie à la campagne, sa description du monde paysan ne vont pas sans équivoque. Les Propos rustiques, comme les Evangiles des Quenouilles, donnent un témoignage exact sur les habitudes et les mentalités populaires. Les historiens, les folkloristes, l'utilisent comme source; ces derniers ravis d'y trouver un tableau concret et diversifié de la culture traditionnelle et de la tradition orale, y pui- 
sent des textes de chansons, des mentions de danses, des descriptions de costumes, des jeux, des coutumes, des dictons. . . . Parlant du peuple, Noël du Faïl dans les Propos rustiques prétend parler comme lui. Son style cherche à reproduire celui des conteurs avec leurs digressions, les interruptions des auditeurs et les traits propres au style parlé: plaisanteries, expressions proverbiales, ton sententieux. ${ }^{47}$ Or une telle fidélité au monde rustique paraît malgré tout un peu suspecte. Le tableau idyllique qu'il en donne manifeste un parti-pris mélioratif révélateur des préjugés de Noël du Faîl, c'est-à-dire de la frange conservatrice de sa classe sociale, la noblesse. Plutôt qu'un éloge des moeurs rurales, il faudrait voir dans les oeuvres de N. du Faïl, un éloge des "états séparés" "48. que chacun reste à sa place, "que le laboureur demeure en la beauté et facilité de ses champs." 49 Il ne s'agit donc pas d'une description du monde, mais d'une vision du monde axée sur une série d'oppositions binaires. A la campagne reliée au naturel, à la transparence, à la sincérité, s'oppose la ville, lieu antinaturel, d'hypocrisie et de calomnie. Le paysan, dans cette division binaire, est valorisé aux côtés de la noblesse, comme pôle positif face à la bourgeoisie, classe montante et menaçante, pôle négatif. ${ }^{50}$

Noël du Faill adopte la veine populaire, comme tant d'autres au XVIe siècle, mais il manifeste une attitude plutôt populiste qui nous permet de mieux apprécier le regard qu'il porte sur les Evangiles des Quenouilles lorsqu'il les considère sous leur aspect documentaire et non sous celui de référence comique. S'il sait encore le leur faire jouer à l'occasion, c'est qu'il n'a pas perdu la notion d'un rire relié aux images du monde à l'envers. Cette façon double de les traiter montre qu'on ne trace plus la coupure entre chaque élément du réel pour en dénier l'univocité, mais qu'on cherche à établir maintenant des catégories univoques et qui s'excluent. L'attitude de Noël du Faill pose en cela des jalons qui nous permettront d'évaluer la position prise par les moralistes à l'égard des Evangiles des Quenouilles.

Pour Pierre de Burry, moraliste et pédagogue, chanoine d'Amiens et précepteur d'enfants de familles nobles, ${ }^{51}$ il ne fait pas de doute que les Evangiles des Quenouilles doivent être pris au sérieux. Ils présentent un danger et c'est à leur contenu de croyances populaires qu'il s'en prend dans un poème contre la superstition ${ }^{52}$ :

Quid ridenda et frivola texo?

Mille alia extant codice multo

Cui nomen muliebria condunt

Arma: colus, teretes quoque fusi.

Comme il ne peut pas éliminer le comique des Evangiles des Quenouilles, il taxe de frivolité un rire maintenant pris en mauvaise part. 
La position d'Olivier Maillard à l'égard des Evangiles des Quenouilles est tout aussi double et contradictoire. D'un côté, il leur accorde assez d'importance pour les trouver dangereux; de l'autre, il les méprise assez pour en faire un synonyme de fable, de sornette, de conte à dormir debout, où il n'y a pas à trouver une once de bon sens. En ce cas, l'irrationnel n'est perçu ni interprété comme élément nécessaire à la cohérence du monde, mais comme pure négativité. "Et ce n'est pas compte ne fable escript au livre des Conoilles," insiste-t-il pour appuyer l'exemplum par lequel il veut montrer, dans son sermon De stipendio peccati, la vraie nature du péché. ${ }^{53}$ Pourtant, audelà de la simple dérision, cette citation des Quenouilles, compte tenu du ton adopté et du contexte de sermon public où elle se trouve insérée, joue un rôle complexe. En la lançant du haut de la chaire, Olivier Maillard nous prouve la popularité de l'ouvrage: il l'utilise pour l'impact qu'il ne manquera pas de produire. Il s'adresse à un public extrêmement diversifié où se mêlent aux étudiants et aux clercs, les bonnes gens du peuple. ${ }^{54}$ Est-ce ceux-ci qu'il vise en citant les Evangiles des Quenouilles, non au sein d'un développement théologique, mais d'une illustration anecdotique? Maillard connaît le langage du peuple et ses expressions familières ou même argotiques, ${ }^{55}$ pourquoi ne lui servirait-il pas un ouvrage auquel il peut s'identifier? Voilà qui porte à s'interroger sur l'attitude du prédicateur à l'égard du public populaire et de la littérature qui est censée le représenter. On peut y voir, et une méconnaissance de la valeur de sa culture, et l'habileté de l'orateur quelque peu bateleur à manipuler les formes d'expression qu'il veut acculturer. Car il arrive que le moraliste quitte le ton patelin et montre les dents pour mieux se faire entendre. Les jeux sont alors clairs: si on ne peut persuader, on convaincra par la force. Et là Maillard ne rit plus des Evangiles des Quenouilles: il les considère comme un des livres pernicieux, "obscènes, traitant de la luxure, de l'art d'aimer, et fournissant une occasion permanente de péché." ${ }^{\prime 56} \mathrm{P}$. de Burry rejetait les Quenouilles parce qu'elles enseignaient les superstititions, Maillard parce qu'elles portent atteinte à la morale. Il n'en reste d'ailleurs pas là et préconise la censure contre toute littérature de ce type, jugée dangereuse: que les évêques appliquent les mesures de contrôle imposées par le pape Innocent VIII.

Voilà qui élargit considérablement le problème. Les Evangiles des Quenouilles se trouvent pris dans une révolution culturelle qui les dépasse de beaucoup, mais dont ils sont aussi le reflet. L'imprimerie arrive, ses techniques se généralisent qui vont assurer le triomphe de l'écrit. Le conflit oral/écrit resté latent au Moyen âge à cause de la précarité des moyens de diffusion du livre, va se trouver ouvert. C'est sous ce jour qu'il faut situer l'oeuvre de Rabelais et l'intérêt si vif de tant d'auteurs du $\mathrm{XVIe}$ siècle pour les formes de l'expressivité orale.$^{57} \mathrm{Ce}$ conflit entre l'oral et l'écrit, Eloy d'Amerval nous le laisse percevoir clairement, justement à propos des Evangiles des Quenouilles, au début de son Livre de la Deablerie. ${ }^{58} \mathrm{Il}$ fulmine contre ceux qui lisent des ouvrages semblables au lieu d'écouter les 
sermons des frères prêcheurs. E. d'Amerval qui fut chef de choeur de l'église sainte Croix à Orléans veut, ${ }^{59}$ en bonne pédagogie, distraire en instruisant, et si la Diablerie s'inscrit dans le genre de la satire des états, elle emprunte plus à l'observation et à la vie quotidienne qu'aux autorités livresques. ${ }^{60}$ Lui aussi va se contredire à propos des Evangiles des Quenouilles. Il leur emprunte des traits de moeurs qui correspondent à des habitudes qu'il connaît et qu'il veut extirper, mais redoute leur mauvaise influence:

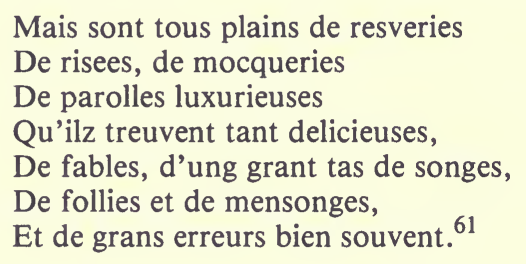

Et ceci, “Combien qu'il ne vault deux grenoilles," dit-il à propos des Quenouilles. Les nécessités de la rime provoquent de bien étranges rencontres! N'empêche, le rejet est catégorique et le terme de mépris vient de lui-même sous la plume de l'auteur qui “'desprise livres imbulles et lubriques non tendans a bonnes moeurs et vertus.' D'Amerval oppose donc deux types de littérature, la bonne et la mauvaise, c'est-à-dire celle qui incite à la sagesse et aide à faire son salut, et la kyrielle des "livres infructueux." A vrai dire, sa distinction ne recoupe pas exactement l'opposition entre profane et sacré. Le divertissement profane trouve grâce à ses yeux lorsqu'il s'agit de la Belle dame sans merci, exemple donné aux femmes de la conduite à tenir et même, étrange indulgence, de François Villon qui "a farcer se delectoit." 62

Les Evangiles des Quenouilles ont cessé d'être l'archétype du comique pour devenir celui de la littérature pernicieuse. On ne les cite plus alors seuls, pour eux-mêmes, mais à l'intérieur de listes d'ouvrages à rejeter comme celle que donne Antoine du Saix dans L'Esperon de discipline, ${ }^{63}$ ' 'doctrinal qui se double d'une véritable encyclopédie sur l'éducation et la culture au début du XVIe siècle." 64

A suivre le fil tissé par les Evangiles des Quenouilles à travers la littérature des XVe et XVIe siècles par les oeuvres qui les ont cités, on se retrouve au coeur du problème posé par l'impact et les conséquences de l'imprimerie, ${ }^{65}$ et dont les contemporains ont pris une conscience précise et quasi-immédiate. Des pédagogues comme P. de Burry, d'Amerval, du Saix, se situent dans la transition entre l'apprentissage par l'acte et la parole transmise d'une part, l'instruction par le livre de l'autre: ils y contribuent en rédigeant des traités d'éducation. Une circulation accrue du livre signifie aussi publicité plus grande accordée au scandale en général, mais plus précisément à l'hérésie, conséquence qui a alerté les réformés 
aussi bien que les catholiques. Et pourtant le livre imprimé ne fournissait-il pas une tribune exceptionnelle à leurs oeuvres pastorales et polémiques, une tribune qui allait jusqu'à abolir les frontières? C'est en effet à la demande de paroisses françaises des Pays-Bas que Calvin rédige son traité contre les Libertins ${ }^{66}$ où sa verve pamphlétaire se plaît à citer les Evangiles des Quenouilles. ${ }^{67}$ Calvin s'y livre à une réfutation systématique, paragraphe par paragraphe, d'un traité d'Antoine Pocques, membre de cette secte "phantastique et furieuse des Libertins" appelée aussi Quintinistes du nom de l'un de ses chefs, Quintin. ${ }^{68}$ Et c'est pour dévaluer sans appel ce que par ailleurs, il taxe de coq à l'âne et d' "amas de sentenses mal cousues," 69 qu'il le compare aux Evangiles des Quenouilles:

Voyez l'audace de ce pendard, de n'avoir nulle honte de contrefaire le grand docteur, en racomptant des fables du Livre des Quenouilles, pour expositions mystiques de l'Escripture. ${ }^{70}$

A l'instar de cet autre grand prêcheur, O. Maillard, par Evangiles des Quenouilles, il entend sottise sans valeur et, comme il le dit pour l'écrit de Pocques, "vessies bien gonflées en apparence et au reste, pleines de vent." 71 Il partage à leur égard l'animosité générale des moralistes contre la littérature de divertissement et s'en explique:

Et ainsi tout ce qui sera mis avant sans aucun fruit et qui ne servira de rien au salut de ceux auxquels on parle, cela est tenu comme fable, ce sont contes qu'on fera pour plaisanter afin de nous faire passer le temps, voire des contes de la cigogne comme on dit. ${ }^{72}$

Mais pour lui, citer les Evangiles des Quenouilles, c'est mettre en oeuvre une stratégie qui procède par insinuations et qui dépasse la dérision, l'arme classique des pamphlétaires. Dans l'immédiat, Calvin voit là un moyen de faire rejaillir sur Pocques, Quintin et leurs émules, le peu d'estime qu'il accorde aux Quenouilles et autres contes de la cigogne, un moyen d'assimiler les Libertins à ces ruraux illettrés que l'ouvrage évoque. Calvin recourra donc à l'insinuation et c'est ce qui nous autorise à trouver dans sa citation des Evangiles des Quenouilles plusieurs autres niveaux de sens. A la lire comme une accusation, celle-ci: les principes des libertins spirituels et le traité de Pocques ne sont que pure superstition. ${ }^{73}$ Or, de superstition, on glisse à satanisme sans y prendre garde. Et l'accusation de satanisme, Calvin la formule en termes à peine voilés à propos de Quintin qui trotte "ça et là pour troubler les povres ames, par tout où il peut sans dangier servir au Diable son maistre."74 La simplicité du rustique, la superstition sinon la sorcellerie, l'hérésie, les Evangiles des Quenouilles portent tout cela en eux, à quoi s'ajoutent des connotations à la licence sexuelle que Calvin 
n'ignorait pas. D'elles aussi, il veut jouer puisqu'il prétend faire la preuve de l'immoralisme des Libertins spirituels et assimiler la liberté de leur conduite sexuelle à celle des bêtes, des "pourceaux," comme il les appelle dans son pamphlet. ${ }^{75}$ Calvin n'est pas sans avoir conscience que les thèmes que charrient les Quenouilles appartiennent au registre comique. Mais son attitude à leur égard est biaisée. Il utilise les images du monde à l'envers comme il le fait pour les proverbes ou les expressions familières, ${ }^{76}$ pour mieux séduire. Loin d'accepter comme inévitable l'envers du juste et de la raison, et de l'intégrer dans son système de pensée, il le met à jour dans le but de l'évacuer. Il participe au processus général d'acculturation auquel procèdent les élites urbaines, protestantes ou catholiques, qui ont décidé de prendre en charge les masses, de les discipliner, de les moraliser.

Les historiens Robert Muchembled et Jean Delumeau ${ }^{77}$ n'ont pas hésité à taxer de répression systématiquement entreprise, le mouvement qui a commencé dans les villes dès le XVe siècle par la dépréciation d'une culture populaire dont on ne perçoit plus la cohérence. On renvoie au sauvage, au superstitieux, au païen ou au satanique, aussi bien les systèmes culturels des communautés traditionnelles que ceux des indigènes des mondes nouveaux que leur récente découverte livre à la pression unificatrice des autorités civiles et religieuses et de leur conception du monde. On va dresser des frontières entre le sacré et le profane, la bonne et la mauvaise littérature, les genres bas et les genres nobles.

Mais à l'époque de l'auteur anonyme qui mettait en forme les Evangiles des Quenouilles, ces distinctions s'opposaient-elles déjà avec netteté? Il faut voir autre chose dans son parti-pris ironique à l'égard des croyances qu'il rapporte et du monde rural et féminin que pure moquerie ou jugement péjoratif. Dans la mesure où il désire, avec les Evangiles des Quenouilles, produire une oeuvre qui appartient au registre comique, il participe à ce "mode dialogique d'être au monde,"78 mode pluriel où coexistent l'affirmation d'une chose et sa négation. L'ironie et la dérision chez lui comme chez les auteurs de farces, les fous ou les participants au Carnaval, créent cette bénéfique ambivalence dont les époques ultérieures ne devaient plus comprendre la fécondité. ${ }^{79}$

\section{Institut D’Etudes Médiévales, Université de Montréal}

\section{Notes}

1 De deux états successifs du texte, témoignent le ms. de Chantilly, musée Condé, 654, pour une première rédaction et le ms. Paris B.N. fr. 2151, pour la version remaniee.

2 Voir le Gesamtkatalog (GW9484).

3 Las ordonansas et coustumas del libre blanc observadas de tota ancianetat, compausadas per las sabias femnas de Tolosa et regidas en forma deguda per lor secretari (816 vers), signées en 
acrostiche à la fin du recueil par Pierre Ducèdre et imprimées à Toulouse par Jacques Colomiès, en 1555.

4 Pour une introduction plus détaillée à cette oeuvre encore si mal connue, je renvoie à l'ouvrage de Gabriel Pérouse, Nouvelles francaises du XVIe siècle. Images de la vie du temps (Genève: Droz, 1977) et plus précisément à son premier chapitre: ' $L$ 'héritage médiéval. Genres littéraires et peintures de vie quotidienne au XVe siècle," p. 16-28.

5 Deuxième journée, chapitre 21. Le texte cité est celui de mon édition critique des Evangiles des Quenouilles: Les "Evangiles des Quenouilles," édition critique et analyse de contenu, thèse de Ph.D. (Sciences médiévales), Université de Montréal, 1980.

6 Edition Pierre le Verdier (Rouen: Société des bibliophiles normands, 1885).

7 Ecrit en marge de la Nativité: “'On placera ici quelque récit pour récréer joyeusement l'esprit des auditeurs"' (d'après Louis Petit de Julleville, Les Mystères [Paris: Hachette, 1880], I, p. 266-67).

8 Mystère de l'Incarnation, éd. Le Verdier, II, p. 248 et suivantes.

9 Viollet le Duc, Ancien thêâtre français (Paris, 1854-57; Réimpr. Neudeln: Krauss), t. II, p. 35-49. Sera cité sous le sigle ATF.

10 Barbara Bowen, Les caractéristiques essentielles de la farce française et leur survivance dans les années 1550-1620 (Urbana: University of Illinois Press, 1963), p. 49. Alan Knight, "The Farce Wife: Myth, Parody and Caricature," dans A Medieval French Miscellany, Norris J. Lacy éd. (University of Kansas Humanistic Studies, 1972), p. 18-19.

11 ATF, II, p. 37.

12 Ne s'introduisent-ils pas ainsi: “Cy commence le traittié intitulé les Euvangiles des Quenoilles faittes a l'onneur et exaucement des dames"?

13 Les maris cocus et souvent consentants, on les trouve dans l'autre catégorie de farces conjugales, celle qui montre des femmes infidèles, à la faim sexuelle insatiable qui les jette d'amants en galants, de gorriers à autres Resjouis d'Amours: Bowen, p. 49; Knight, p. 18-19.

14 Konrad Schoell, “Des farces féministes?" dans Gari R. Muller, Le théâtre au Moyen âge, (Montréal: L'Aurore, 1981), p. 169-85.

15 Halina Lewicka, "La farce médiévale était-elle un genre populaire?" Travaux de linguistique et de littérature, 16 (1978), p. 335-41.

16 Pierre de Larrivey, Les Tromperies, ATF, VII, p. 5-105 (acte II, scène 2).

17 Ibid., acte II, scène 2, p. 34 .

18 Sur la fortune de La Célestine en France, Gustave Reynier. Les origines du roman réaliste (Paris, 1922; réimpr. Genève: Slatkine, 1969); Gérard J. Brault, Celestine. A critical Edition of the first French Translation (1527) of the Spanish Classic "La Celestina" with an introduction and notes (Detroit: Wayne State University Press, 1963); Krystyna Kasprzik, Nicolas de Troyes et le genre narratif en Grance au XVIe siècle (Paris: Klincksieck, 1963).

19 Nicole Moine, “Augustin et Apulée sur la magie des femmes d'auberge," Latomus, 34 (1975), p. 350-361. Sur le type de l'entremetteuse, voir pour le Moyen âge: Félix Lecoy, Recherches sur le "Libro de buen Amor" (Paris: Droz, 1938) et pour les époques ultérieures, Léon et Frédéric Saisset, "L'entremetteuse," Mercure de France, 15 (1922), p. 111-129; Jacques Bailbé, "Le thème de la vieille femme dans la poésie satirique du XVIe et du début du XVIle siècle," Bibliothèque d'humanisme et de Renaissance, 26 (1964), p. 98-119; L. Guillern-Curutchet, J.P. Guillern, L. Hordoir-Louppe, M. FR. Piejès, La femme dans la littérature française et les traductions en français du XVIe siècle (Lille: Université de Lille, 1971); Madeleine Lazard, La comédie humaniste et ses personnages (Paris: P.U.F., 1978).

20 Prologue de la troisième soirée.

21 Mikhaill Bakhtine, L'oeuvre de François Rabelais et la culture populaire au Moyen âge et sous la Renaissance (Paris: Gallimard, 1970), p. 240.

22 ATF, II, p. 207-222 (Citation p. 208).

23 ATF, II, p. 140-157.

24 ATF, II, p. 152.

25 Petit de Julleville, Les Mystères, I, p. 231.

26 Edition J. Carnaudet (Paris: Techener, 1855), p. 43.

27 Bakhtine, L'oeuvre de François Rabelais, p. 16. 
28 Dreux du Radier, Récréations historiques, critiques, morales et d'érudition, avec l'histoire des fous en titre d'office (Paris: 1762): “Toute sa science était tirée d'un ancien recueil de contes intitulé Les Evangiles des Quenouilles, faits et racomptez par plusieurs notables Dames." Les Evangiles des Quenouilles n'apparaissent pas dans la liste des ouvrages de la bibliothèque de Maître Guillaume, dont l'inventaire a été publié: Inventaire des livres trouvez en la bibliothèque de Maître Guillaume (Cologne, 1739).

29 Paul Zumthor, Le Masque et la Lumière (Paris: Le Seuil, 1978), p. 130.

30 Bakhtine, L'oeuvre de François Rabelais, p. 24.

31 Et qui peuvent tourner mal, comme cette "montre" que raconte Rabelais au chapitre XIII du Quart Livre.

32 Jean-Claude Aubailly, Le monologue, le dialogue et la sottie, Essai sur quelques genres dramatiques de la fin du Moyen âge et du début du XVIe siècle (Paris: Champion, 1976), p. 252-53; Moshé Lazar, "Les diables: serviteurs et bouffons," Tréteaux, I (1978), p. 51-69 (p. 63).

33 Bakhtine, L'oeuvre de François Rabelais, p. 27.

34 Zumthor, Le masque, p. 130.

35 Jean Molinet, Les faictz et dictz, éd. Noël Dupire (Paris: Droz, 1937), p. 915-17.

36 Pierre Champion, Histoire poétique du quinzième siècle (Paris: Champion, 1923), p. 386.

37 Il s'agit de rhétorique et non d'obsession sexuelle, d'érotomanie comme le déplore Omer Jodogne dans "Le caractère de Jean Molinet," La Renaissance dans les provinces du Nord (Paris: C.N.R.S. 1956), p. 97-111.

38 Voir Dupire, p. 301. Le titre de la lettre donne un exemple de ces constructions lexicales qui ne sont pas sans faire penser à Rabelais; ainsi à la ligne 7, il s'adresse à "vostre superlicoquence venerabilitudinicque chinocrochitrorieusement."

39 Un exemple: "Vous chantés tout seul et tout ensamble ung duo a trois du neufviesme ton" (p. 916).

40 Jean Molinet, Les faictz et dictz, éd. citée, p. 916.

41 Rabelais, Quart Livre, chap. LX.

42 Noël du Faïl, Contes et discours d'Eutrapel, éd. J. Assézat (Paris: Daffès, 1874).

43 Ibid., II, p. 137.

44 Noël du Faïl, Propos rustiques, éd. R. Lefèvre (Paris: Garnier, 1928), p. 39; Ev. des Quenouilles: ligne 148. Voir sur ce terme commun, Emmanuel Plilipot, La vie et l'oeuvre littéraire de Noël du Fail, gentilhomme breton (Paris: Champion, 1914), p. 104.

45 Propos rustiques, éd. citée, p. 95-96.

46 Contes d'Eutrapel, éd. citée, II, p. 259.

47 Noël du Faïl, Les baliverneries d'Eutrapel, éd. Gaël Milin (Rennes: Université de Rennes, 1969), p. xcv.

48 Olivier Martin, "Noël du Faïl et le rôle actuel de la noblesse," Mémoires de la société d'histoire et d'archéologie de Bretagne, 8 (1927), p. 257-76.

49 Contes d'Eutrapel, éd. citée, II, p. 232.

50 Gaël Milin, "Modèles idéologiques et modèles culturels dans l'oeuvre narrative de Noël du Faïl," Annales de Bretagne, 81 (1974), p. 65-104; les divisions temporelles s'intègrent à ce système: passé, du côté positif/présent, du côté négatif.

51 Ferdinand van der Haeghen, Bibliotheca Belgica, rééd. M. TH. Leuger (Bruxelles, 1964), t. I, p. 388-94.

52 Moralium Magistri Petri Burri Carminarum libri novem cum argumentis et vocabulorum minus vulgarium compendiosa explanatione, imprimé à Paris, le 20 mars 1503 . La citation est au f. xxiiij.

53 Sermo Secundus (Lyon: Geygnard, 1503), f. $\operatorname{cccxx}^{\mathrm{r}}$, col. 2 (B.N. Rés. D 5197). L'exemplum raconte l'histoire de Thamar (2.5à 13) et fustige ces "macqueraulx" (en français dans le texte), poissons de mer, mais aussi ces maris qui vendent leurs femmes, ces pères ou mères qui vendent leur fille.

54 J.P.A. Samouillan, Olivier Maillard, sa prédication et son temps (Paris, Bordeaux, 1891), p. 75; 
Bernard Chevalier, "Olivier Maillard et la réforme des Cordeliers, 1482-1502," Revue de l'histoire de l'Eglise de France, 65 (Janv-juin 1979), p. 25-39.

55 Tel l'emploi du terme “maquereau" (voir note 52).

56 Traduction de Samouillan, p. 277. Extrait du sermon prononcé pour le carême de saint Jean en Grève en 1494.

57 Bruno Roy et Madeleine Jeay, “L'émergence du folklore dans la littérature du XVe siècle," dans Fifteenth Century Studies, 2 (1979), p. 95-113.

58 Eloy d'Amerval, Le livre de la Deablerie, chez l'imprimeur Michel le Noir, Paris, 1508. Voir à propos de l'édition moderne de cette oeuvre par Charles F. Ward (Iowa: University of Iowa Press, 1922), le compte-rendu très critique de Mario Roques, Romania, 49 (1923), p. 473-74.

59 Michel Brenet, “Un poète-musicien français du XVe siècle, Eloy d'Amerval," Congrès international d'histoire de la musique, Solesmes, 1901, p. 165-172.

60 Winthrop H. Rice, Le "livre de la Deablerie," Cahiers de l'association internationale des études françaises, 1953, p. 115-126; Jill Hann, Chaucer and the Medieval Estates Satire. The Literature of Social Classes and the General Prologue of the "Canterbury Tales" (Cambridge: Cambridge University Press, 1973).

61 Livre 1, chap. xxxix (éd. Ward, p. 40). Les citations suivantes sont tirées du même passage.

62 Liv. II, chap. liij (p. 103), et liv. II, chap. .lxiij (p. 118).

63 L'esperon de discipline pour inciter les humains aux bonnes lettres, stimuler a doctrine, animer a science et inviter a toutes bonnes oeuvres vertueuses et morales, par consequent pour les faire coheritiers de Jesus Christ et expressement les nobles et genereux. Lourdement forgé et rudement limé par noble homme fraire Antoine du Saix, Commandeur de Sainct Antoine de Bourg en Bresse, Paris, 1532.

64 Ch. Béné, "Antoine du Saix, humaniste et émule de Rabelais," Actes de colloque sur l'humanisme lyonnais au XVle siècle, mai 1972 (Grenoble: Presses universitaires de Grenoble, 1974), p. 245-60.

65 Elisabeth L. Eisenstein, The Printing Press as an Agent of Change (Cambridge: Cambridge University Press, 1979).

66 Jean-Claude Margolin, “'Libertins, libertinisme et 'libertinage' au XVIe siècle" dans Aspects du libertinisme au XVIe siècle (Paris: Vrin, 1974), p. 1-33.

$67 \mathrm{~J}$. Calvin, Contre la secte phantastique et furieuse des Libertins qui se nomment spirituelz, dans Calvini Opera, vol. VII (Corpus Reformatorum, t. 35), col. 149-248.

68 Margolin, p. 11; Frantiśek Bartoś, "Picards et Pikarti," Bulletin de la societé d'histoire du protestantisme français, 80 (1931), p. 465-86.

69 Calvin, Contre la secte, éd. citée, col. 241.

70 Ibid., col. 229.

71 Ibid., col. 228.

72 D'après Rodolphe Peter, "Rhétorique et prédication selon Calvin." Revue d'histoire et philosophie religieuses, 55 (1975), p. 249-272. E. Pasquier pense-t-il aussi à nos Evangiles des Quenouilles, lorsqu'il s'en prend, dans le même esprit de dérision, à ces discours qui “sont vrayement ce que nous disons en commun Proverbe, contes de la quenouille" (E. Pasquier, Le catéchisme des Jésuites, Villefranche, La Rochelle: Guillaume Grenier, 1602, f. 98v.)? Je remercie Monsieur Claude Sutto pour cette information.

73 A propos de l'extension du concept de superstition sous la plume des réformés: Jean Delumeau, "La lutte du protestantisme contre les superstititions," dans La mort des pays de Cocagne (Paris: Université de Paris I, 1976), p. 97-113.

74 Calvin, Contre la secte, éd. citée, col. 241.

75 Ibid., col. 229.

76 Emile Doumergue, Le caractère de Calvin (Paris, 1921; réimpr. Genève: Slatkine, 1970), p. 34-37, remarque que Calvin aime utiliser le langage familier et ne recule pas devant des termes orduriers. Voir surtout Edmond Huguet, "La langue familière chez Calvin," Revue d'histoire littéraire de la France, 23 (1916), p. 27-52. 


\section{2 / Renaissance and Reformation}

77 Robert Muchembled, Culture populaire et culture des élites (Paris: Flammarion, 1978) et Jean Delumeau, La peur en Occident (Paris: Fayard, 1978).

78 Zumthor, Le masque, p. 126.

79 Un écho affaibli de l'appartenance des Evangiles des Quenouilles au monde du comique nous parvient à la fin du siècle avec le mémorialiste protestant Pierre de l'Estoile qui sait encore les insérer dans un coq-à-l'âne, ceci en compagnie de Rabelais: "As-tu point vu dans Rabelais, /Le cruel combat des Andouilles?/J'ay leu, au Livre des Quenouilles,/Qu'on peult un Roy déposséder/S'il lui plaist ainsi l'accorder/Se mettant lui-même en tutelle (Pierre de l'Estoile, Mémoires-Journaux, éd. G. Brunet, Champollion, Halphen, P. Lacroix, Ch. Read, Tamirey de Larroque, Tricotel [Paris: A Lemerre, 188-96], t. II, p. 268-69). 